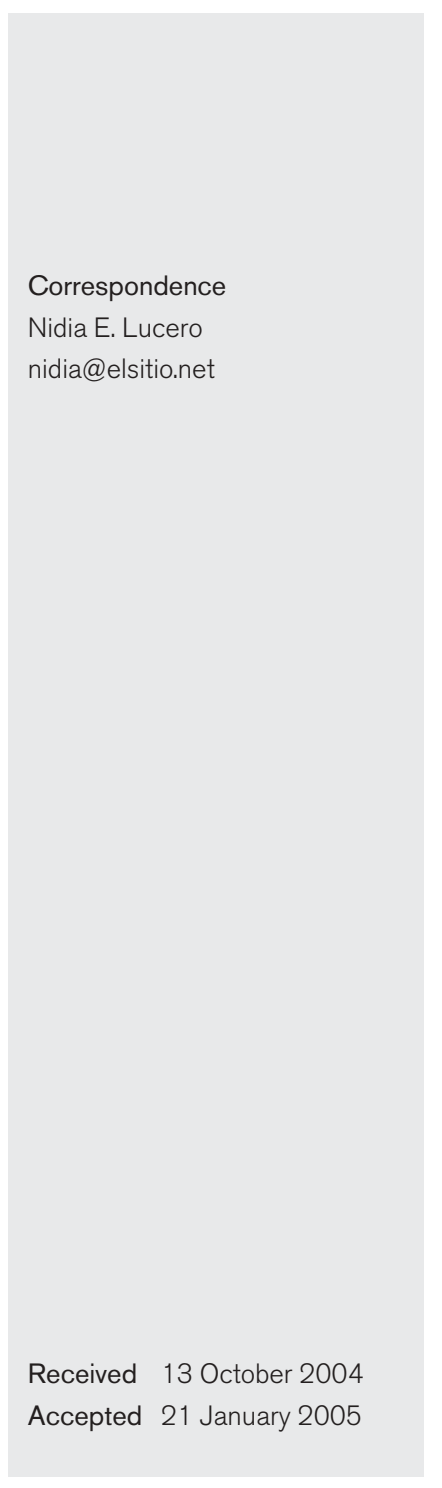

\section{Diagnosis of human brucellosis caused by Brucella} canis

\author{
Nidia E. Lucero, ${ }^{1}$ Gabriela I. Escobar, ${ }^{1}$ Sandra M. Ayala ${ }^{1}$ and Nestor Jacob ${ }^{2}$ \\ ${ }^{1}$ Brucellosis Laboratory, ANLIS Dr C. G. Malbrán, Avda. Velez Sarsfield 563, 1281 Buenos Aires, \\ Argentina \\ ${ }^{2}$ Infectología, Hospital Cosme Argerich, Alte. Brown 240, 1155 Buenos Aires, Argentina
}

\section{INTRODUCTION}

The most common modes of transmission of Brucella canis to man are through contact with infected dogs, which may disseminate the disease for many months after bacteraemia has ceased, through contact with their secretions and through direct laboratory exposure (Carmichael \& Shin, 1996).

The disease is underdiagnosed due to general lack of serological testing facilities and misconceptions concerning its prevalence. Culture-positive cases have been reported in laboratory personnel, animal technicians and persons known to have close and frequent contact with infected dogs (Carmichael et al., 1980).

Abbreviations: BPAT, buffered plate agglutination test; CELISA, competitive ELISA; CF, complement fixation; IELISA, indirect ELISA; RSAT, rapid slide agglutination test; TAT, tube agglutination test.
Human infections are probably more common than indicated in published reports, though serological methods and criteria for evaluating results vary greatly. The serological techniques most often used to detect $B$. canis antibodies in humans are the agglutination tests (Lewis \& Anderson, 1973; Hoff \& Schneider, 1975; Hoff \& Nichols, 1974; Monroe et al., 1975; Flores-Castro \& Segura, 1976; Ying et al., 1999; Polt \& Schaefer, 1982). The infection was diagnosed by serological methods in a 17-month-old child, a woman with fever of unknown origin and a man with granulomatous hepatitis and splenomegaly (Tosi \& Nelson, 1982; Rousseau, 1985; Schoenemann et al., 1986). Complications such as mycotic aneurysms of the tibioperoneal arteries, aortic valve vegetations, calvarial osteomyelitis and more recently a presumptive case of B. canis endocarditis, diagnosed by serology at the Centers for Disease Control and Prevention (Atlanta), have been described (McKee \& Ballard, 1999; Ying et al., 1999; Piampiano et al., 2000). 
As a national centre for human brucellosis our laboratory is engaged in the serological and bacteriological diagnosis of patients with symptoms and/or epidemiology compatible with this disease. On the basis that people could potentially be infected by B. canis we used serological and bacteriological methods to study patients with negative serological tests to smooth-Brucella abortus antigen.

Recently we reported an indirect ELISA (IELISA) test for the detection of antibodies to $B$. canis in dogs that has been demonstrated to be highly specific and sensitive (Lucero et al., 2002). We now report the potential use of this IELISA for the diagnosis of human brucellosis caused by B. canis in a population of patients who tested positive by rapid slide agglutination test (RSAT) but negative by smooth $B$. abortus antigen.

\section{METHODS}

Human sera. Sera from the 179 people included in the study were classified into five groups. The first group consisted of 17 sera from patients with positive B. canis culture or in close contact with culturepositive dogs. The second group of 110 sera was obtained from asymptomatic people with no clinical or epidemiological evidence of brucellosis, with negative blood culture and negative RSAT, buffered plate agglutination test (BPAT), tube agglutination test (TAT), complement fixation (CF) and competitive ELISA (CELISA) results. A third group, suspected of having brucellosis caused by B. canis, included 35 patients with clinical symptoms compatible with brucellosis and negative BPAT, TAT, CF, CELISA tests and blood culture but positive or weakly positive RSAT results. The fourth group included nine sera from patients with infectious diseases other than brucellosis supplied by the Bacteriology Department, INEI-ANLIS 'Dr C. G. Malbrán'. Another eight sera were from patients with brucellosis caused by smoothBrucella species isolated and typed at our laboratory.

Serological tests. BPAT, TAT and CF were run as described previously (Lucero \& Bolpe, 1998) with antigens prepared at ANLIS 'Dr C. G. Malbrán' using the B. abortus 1119-3 strain. CELISAs were run as previously reported (Lucero et al., 1999); the antigen (S-LPS from $B$. abortus 1119-3) and the MAb were standardized and supplied by the Brucellosis Centre of Expertise and OIE Reference Laboratory, Animal Diseases Research Institute (ADRI), Canada. The conjugate preadsorbed with bovine, equine and human serum protein was from Jackson Lab.

RSAT. The RSAT was used as a screening test, run as described previously (Lucero et al., 2002; Carmichael \& Joubert, 1987) with serial sera dilutions in order to find the final titre. Briefly, $10 \mu \mathrm{l}$ of serum dilution was mixed with $10 \mu \mathrm{l}$ of antigen on a $25 \times 75 \mathrm{~mm}$ glass slide for $1-2 \mathrm{~min}$ and the results were read under a $\times 10$ microscope objective. A strong control serum with a known titre was also included. The 2mercaptoethanol (2ME)-RSAT was performed by mixing $25 \mu \mathrm{l}$ of serum dilution with $25 \mu \mathrm{l}$ of $0 \cdot 2 \mathrm{M}$ 2-ME solution; after 1 min $50 \mu \mathrm{l}$ of antigen was added and read in the same way. The antigen was prepared at ANLIS 'Dr C. G. Malbrán' with the strain $(\mathrm{M}-)$ variant of $B$. canis.

IELISA. The antigen was obtained from the $(\mathrm{M}-)$ variant of $B$. canis as described previously (Lucero et al., 2002). Briefly, B. canis hot saline extract was prepared, then centrifuged at $254000 \mathrm{~g}$ in a Kontron Instrument UltraCentrifuge in a TFT 45.94 rotor for $4 \mathrm{~h}$ at $4{ }^{\circ} \mathrm{C}$. The pellet was dissolved in PBS, pH $7 \cdot 2$, frozen at $-20^{\circ} \mathrm{C}$ and used at a $1: 2000$ dilution after $\mathrm{OD}_{414}$ readings of various antigen dilutions using strongly positive, weakly positive and negative sera as controls. The strong control sera were from a patient who had positive haemoculture and a positive RSAT with a titre of $1: 16$, and the weakly positive control sera were from a patient who had a positive RSAT with a titre of $1: 2$ and negative haemoculture. The negative serum was from a healthy person with negative haemoculture and serological tests for both smooth and rough antigens.

A lyophilized horseradish peroxidase-conjugated protein A/G was from ImmunoPure (Pierce Lb.) and was used at 1:20 000 after testing various working dilution ranges with strongly positive, weakly positive and negative human sera.

The antigen diluted in 0.06 $\mathrm{M}$ sodium carbonate buffer ( $\mathrm{pH} 9 \cdot 6$ ) was passively coated onto polystyrene plates (Nunc 2-69620, Denmark) at $50 \mu \mathrm{l}$ per well, incubated for $18 \mathrm{~h}$ at room temperature and then washed five times in 0.01 M PBS containing 0.05\% Tween 20, pH 7.2 (PBS/T). Control and test sera were added at $1: 50$ in PBS/T, 50 ul per well, for $1 \mathrm{~h}$ at room temperature. After five washes in $\mathrm{PBS} / \mathrm{T}$, appropriately diluted horseradish peroxidase-conjugated protein $\mathrm{A} / \mathrm{G}$ was added, $50 \mu \mathrm{l}$ per well, and incubated for $1 \mathrm{~h}$ at room temperature. After five washes in $\mathrm{PBS} / \mathrm{T}$, the final step was the addition of $100 \mu \mathrm{l}$ per well of chromogenic substrate $\left(4.0 \mathrm{mM}\right.$ hydrogen peroxide and $1.0 \mathrm{mM} 2,2^{\prime}$-azino-bis(3ethylbenz-thiazoline-6-sulfonic acid) diammonium salt in $0.05 \mathrm{M}$ citrate buffer, $\mathrm{pH} 4 \cdot 5)$. The plate was shaken continuously on an orbital shaker and after 10 min the $\mathrm{OD}_{414}$ was measured in a photometer (Labsystems Multiskan EX microplate reader) with $100 \mu \mathrm{l}$ of chromogenic substrate in a plate as a control for the microplate reader. The test is positive when colour develops. The standard control serum used on each plate makes it possible to convert the optical density reading to percent positivity $(\% \mathrm{P})$.

Bacteriological studies. Brucella organisms were isolated from three human blood cultures by inoculating $5 \mathrm{ml}$ of blood into $25 \mathrm{ml}$ of liquid medium. Only one blood culture was done on serum from each dog using paediatric bottle holding. The strains isolated were typed as recommended by the former ICBN Subcommittee on Taxonomy of the Genus Brucella (Corbel \& Brinley-Morgan, 1984) at ANLIS 'Dr C. G. Malbrán'.

Data analysis. The strong control sera were from a patient who had positive haemoculture and positive RSAT with a titre of $1: 16 . \mathrm{OD}_{414}$ values from the IELISAs were compared to those obtained with the strong control serum included in each 96-well plate and a relative percent positivity value $(\% \mathrm{P})$ was calculated as follows (Nielsen et al., 2004): $\% \mathrm{P}=\left(\mathrm{OD}_{414}\right.$ of test sample/ $\mathrm{OD}_{414}$ of strong control serum $)$ $\times 100$.

Diagnostic specificity and sensitivity were determined initially with $95 \%$ confidence limits by plotting the data for negative and positive samples on a frequency histogram. Data were subsequently analysed by receiver-operator characteristics analysis (Schoonjans et al., 1995).

\section{RESULTS AND DISCUSSION}

We used RSAT as a screening test to study patients with symptoms and/or epidemiology compatible with brucellosis but negative results to tests with smooth-B. abortus antigen. Another objective was to ascertain the usefulness of an IELISA as a confirmatory test and to determine the cut-off value.

The serological study was run on 179 sera. One hundred and ten sera from healthy people were examined and found to be negative by tests using smooth B. abortus antigen (BPA, TAT, CF and CELISA) and RSAT (B. canis $\mathrm{M}$ - antigen), and when 
tested with IELISA showed a mean $\% \mathrm{P}$ value of 16 and a standard deviation (SD) of 5.25. Fig. 1 shows the frequency distribution of these sera. Therefore a cut-off value of $26 \cdot 5$ $\% \mathrm{P}($ mean $+2 \mathrm{SD})$ was established and then adjusted to $27 \% \mathrm{P}$ by receiver-operator characteristic curve using both positive and negative serum samples, resulting in $100 \%$ sensitivity and specificity.

Table 1 shows the serological test results for the 17 sera from patients with positive $B$. canis culture or with close contact with culture-positive dogs, at the time that they came to our

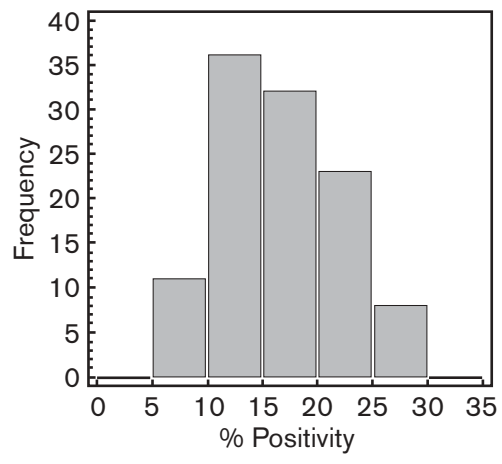

Fig. 1. Frequency distribution of IELISA results from 110 serum samples negative for antibodies to B. canis. The cut-off value of $27 \% \mathrm{P}$ was selected to provide an assay specificity value of $100 \%$. laboratory. IELISA sensitivity was $100 \%$ with a cut-off value of $27 \% \mathrm{P}$. Most of these patients presented clinical symptoms similar to brucellosis caused by other species of Brucella such as fever, asthenia and hepatosplenomegaly. Some of them received antibiotic treatment and made good progress. The serological follow-up performed on serial serum samples from patients who received treatment showed that the RSAT and IELISA tests correlated well with clinical progress. The boy aged 13, who spent 2 weeks in hospital with intermittent fever and hepatosplenomegaly as prominent signs, recovered normally without treatment and was clinically symptom-free 3 months afterwards, with RSAT and IELISA titres declining slowly. Two dogs in contact with the patient were serologically examined, and both gave RSAT-positive results, though only the female (which had four times given birth to weak pups that subsequently died) was IELISA positive.

In the group of 35 patients suspected of having brucellosis (with negative blood culture but positive RSAT), of the 25 that came to our laboratory only once, 13 were positive by IELISA, with RSAT titres that ranged from weak reaction with pure serum to dilution $1: 4$ (data not shown). Of the eight patients that came twice, three were IELISA-positive, with RSAT titres ranging from weak reaction with pure serum to dilution $1: 32$. The two patients that came to the laboratory four and five times were RSAT- and IELISApositive. The 18 RSAT and IELISA-positive cases were from 12 men (age range, 26-61) and six women (age range, 23-

Table 1. Serological response of sera from patients $B$. canis culture-positive or with close contact with $B$. canis-culture-positive dogs

B. canis culture-positive dogs were those that were BPA-, TAT-, CF- and CELISA-negative.

\begin{tabular}{|ccccl|}
\hline Patient & Age $^{*}$ (years) & RSAT $\dagger$ & IELISA (\%P) $\neq$ & \multicolumn{1}{c|}{ Epidemiological data } \\
\hline 1 & 13 & 4 & 100 & B. canis-positive dog owner \\
2 & 31 & 16 & 64 & B. canis culture-positive \\
3 & 26 & Pos $+/-\$$ & 36 & B. canis-positive dog handler \\
4 & 39 & Pos $+/-\$$ & 59 & B. canis-positive dog owner \\
5 & 16 & 32 & 96 & B. canis culture-positive \\
6 & 31 & Neg & 59 & B. canis-positive dog owner \\
7 & 58 & Pos & 29 & B. canis-positive dog owner \\
8 & 10 & Neg & 28 & B. canis-positive dog owner \\
9 & $\mathrm{ND}$ & 32 & 100 & B. canis-positive dog handler \\
10 & 30 & $\mathrm{Neg}$ & 41 & B. canis-positive dog owner \\
11 & 35 & $\mathrm{Neg}$ & 33 & B. canis-positive dog owner \\
12 & 44 & $\mathrm{Neg}$ & 37 & B. canis-positive dog owner \\
13 & 16 & $\mathrm{Neg}$ & 33 & B. canis-positive dog owner \\
14 & 40 & 2 & 35 & B. canis-positive dog owner \\
15 & 13 & $\mathrm{Neg}$ & 35 & B. canis-positive dog owner \\
16 & 23 & Pos & 28 & B. canis-positive dog owner \\
17 & 48 & 2 & 78 & B. canis culture-positive \\
\hline
\end{tabular}

${ }^{\star} \mathrm{ND}$, No data.

$\dagger$ Reciprocal titres.

‡IELISA cut-off, $27 \%$ P.

§Weakly positive. 
88). One was a veterinarian, two were owners of infected dogs, 10 worked with domestic animals and five presented ambiguous epidemiological information. Of the 17 IELISAnegative sera, two were RSAT-positive at dilution $1: 2$ and 15 were weakly positive with pure serum; with 2ME-RSAT, 11 tested negative and six tested weakly positive (data not shown). These sera were probably from patients at an early stage of the infection or indicated false-positive results.

Cross-reactions between Brucella species and other microorganisms that share antigenic determinants causing false-positive reactions have been reported (Corbel, 1985). Similarities in the O-polysaccharide chemical structure of various micro-organisms, such as Escherichia coli O157 : H7, Francisella tularensis, Vibrio cholerae, Salmonella group N and Pseudomonas maltophilia, are responsible for most observed cross-reactions (Nielsen et al., 2004). But no cross-reaction was observed to RSAT or to IELISA in the nine sera studied from patients with infectious diseases other than brucellosis (two with meningitis, two with haemolytic uraemic syndrome, two with salmonellosis, two with diarrhoeas and one with bloody diarrhoea), as shown in Table 2.

However, cross-reactivity was evident in sera from eight patients who had positive haemocultures of B. abortus biovar 1, Brucella melitensis biovar 1 or Brucella suis biovar 1 . This shows that RSAT and IELISA antigens prepared with $B$. canis $\mathrm{M}-$ strain have LPS determinants specific for $B$. canis as well as other antigenic components shared with rough- and smooth-Brucella strains. Cases 1 and 2 (Table 3) were positive to both tests 17 and 45 months after admission, respectively, while case 3 , from whom $B$. abortus biovar 2 was isolated, tested RSAT- and IELISA-negative at admission and 2 months later. Case 4 was negative 3 months after admission, and case 5 continued to be positive 5 months later, while the last three cases with $B$. suis haemocultures presented high titres by RSAT and IELISA. Case 8 was a dog breeder whose dogs presented clinical symptoms of brucellosis and were serology-positive with smooth-Brucella antigen and negative with rough-Brucella antigens, but the dogs' haemocultures were negative, probably because they had received antibiotic therapy.

Although B. canis is recognized as the aetiological agent of an infection in humans and dogs, available information on its prevalence is limited. Several serological surveys have been performed using the TAT in selected population groups: a

Table 2. Serological response of sera from patients with diagnoses other than brucellosis

\begin{tabular}{|lcccll|}
\hline Patient & Age $^{*}$ & RSAT & IELISA (\%P) $\dagger$ & \multicolumn{1}{c|}{ Diagnosis } & Strain isolated \\
\hline 1 & $53 \mathrm{y}$ & $\mathrm{Neg}$ & 23 & Meningitis & Neisseria meningitidis \\
2 & $56 \mathrm{y}$ & $\mathrm{Neg}$ & 22 & Meningitis & E. coli O157 \\
3 & $10 \mathrm{~m}$ & $\mathrm{Neg}$ & 13 & Haemolytic uraemic syndrome & E. coli O157 \\
4 & $\mathrm{ND}$ & $\mathrm{Neg}$ & 18 & Bloody diarrhoea & E. coli O157 \\
5 & $\mathrm{ND}$ & $\mathrm{Neg}$ & 19 & Haemolytic uraemic syndrome & E. coli O157 \\
6 & $2 \mathrm{y}$ & $\mathrm{Neg}$ & 13 & Diarrhoea & E. coli O157 \\
7 & $2 \mathrm{y} 9 \mathrm{~m}$ & $\mathrm{Neg}$ & 9 & Diarrhoea & \\
8 & $40 \mathrm{y}$ & $\mathrm{Neg}$ & 21 & Salmonellosis (Widal positive H: $160 \mathrm{O}: 80$ ) & \\
9 & $\mathrm{ND}$ & $\mathrm{Neg}$ & 26 & Salmonellosis (Widal positive H:80 O :360) & \\
\hline
\end{tabular}

${ }^{*} \mathrm{ND}$, No data; m, months; $y$, years.

$\dagger$ IELISA cut-off, $27 \% \mathrm{P}$.

Table 3. Serological response of sera from patients with brucellosis caused by smooth Brucella species

\begin{tabular}{|lcrrrrrr|}
\hline Patient & BPA & TAT & CF $^{*}$ & CELISA (\%I) $\dagger$ & RSAT $^{*}$ & IELISA (\%P) $^{\text {Species/biovar }}$ \\
\hline 1 & Pos & 1600 & 320 & 77 & 512 & 96 & B. abortus 1 \\
2 & Pos & 400 & 80 & 67 & 64 & B. abortus 1 \\
3 & Pos & 1600 & 40 & 65 & Neg & 25 & B. abortus 2 \\
4 & Pos & 800 & 40 & 52 & Pos & 49 & B. melitensis 1 \\
5 & Pos & 800 & 320 & 74 & Pos & 90 & B. melitensis 1 \\
6 & Pos & 6400 & 640 & 69 & 64 & 100 & B. suis 1 \\
7 & Pos & 400 & 640 & 83 & 32 & 100 & B. suis 1 \\
8 & Pos & 400 & 160 & 93 & 4 & 96 & B. suis 1 \\
\hline
\end{tabular}

\footnotetext{
${ }^{\star}$ Reciprocal titres.
}

$\dagger$ CELISA cut-off, $28 \% \mathrm{I}$.

$\ddagger$ IELISA cut-off, $27 \% \mathrm{P}$. 
study of hospital patients with various complaints in Mexico revealed a $13 \%$ prevalence of significant antibody titres (Flores-Castro \& Segura, 1976); in US military populations $0.4 \%$ positive reactions were detected (Lewis \& Anderson, 1973); 0.59\% in Florida residents (Hoff \& Nichols, 1974; Hoff \& Schneider, 1975) and 67.8 \% in Oklahoma (Monroe et al., 1975); an investigation in Germany found antibody titres in 6 out of 1915 sera (Carmichael et al., 1980). Another study found 21 out of 1065 people to be positive to $B$. canis antibodies by the gel-diffusion test using $B$. ovis antigen (Varela-Diaz \& Myers, 1979) and a microagglutination test using a safranin-dyed B. canis antigen detected the infection in four patients with febrile illness (Polt \& Schaefer, 1982). For TATs using rough-Brucella antigens, the difficulty of establishing a cut-off point and the significant agglutinin titres from non-specific reactions has been recognized (Carmichael et al., 1980).

Understanding that patients showing symptoms compatible with brucellosis could potentially be infected by B. canis, we recommend the use of RSAT and IELISA tests to check sera from cases with negative serological tests to smooth-Brucella antigen.

It is generally agreed that available evidence suggests a low incidence of clinical and subclinical human brucellosis due to B. canis. But it has been emphasized that routine brucellosis diagnosis does not include $B$. canis investigation so infection with this Brucella species may be more widespread than is now suspected. After studying a larger number of samples the RSAT could provide a suitable screening test for the diagnosis of $B$. canis human brucellosis, while a supplementary technique such as IELISA performed on all positive RSAT samples could ensure diagnostic specificity and confirm the diagnosis.

\section{ACKNOWLEDGEMENTS}

We are very grateful to Dr Isabel Chinen at Laboratorio de Fisiopatogenia, Departamento de Bacteriología, INEI-ANLIS 'Dr C. G. Malbrán', who kindly supplied sera from patients with infectious diseases other than brucellosis, and to Dr Klaus Nielsen from the Canadian Food Inspection Agency, Animal Research Institute, Ontario, Canada, for reviewing the manuscript.

\section{REFERENCES}

Carmichael, L. L. \& Joubert, J. C. (1987). A rapid slide agglutination test for the serodiagnosis of Brucella canis infection that employs a variant $(\mathrm{M}-)$ organism as antigen. Cornell Vet 77, 3-12.

Carmichael, L. E. \& Shin, S. J. (1996). Canine brucellosis: a diagnostician's dilemma. Semin Vet Med Surg (Small Anim) 11, 161-165.

Carmichael, L. E., Flores-Castro, R. \& Zoha, S. (1980). Brucellosis caused by Brucella canis: an Update of Infection in Animals and in Humans. Geneva: World Health Organization. Document WHO/ BRUC./80·361 WHO/ZOON./80·135.
Corbel, M. J. (1985). Recent advances in the study of Brucella antigens and serological cross reactions. Vet Bull 55, 927-942.

Corbel, M. J. \& Brinley-Morgan, W. J. (1984). Genus Brucella, Meyer and Shaw 1920, 173 ${ }^{\mathrm{AL}}$. In Bergey's Manual of Systematic Bacteriology, vol. 1, pp. 377-388. Edited by N. R. Krieg \& J. G. Holt. Baltimore: Williams \& Wilkins.

Flores-Castro, R. \& Segura, R. (1976). A serological and bacteriological survey of canine brucellosis in Mexico. Cornell Vet 66, 347-352.

Hoff, G. L. \& Nichols, J. B. (1974). Canine brucellosis in Florida: serological survey of pound dogs, animal shelter workers and veterinarians. Am J Epidemiol 100, 35-38.

Hoff, G. L. \& Schneider, N. J. (1975). Serologic survey for agglutinins to Brucella canis in Florida residents. Am J Trop Med Hyg 24, 157-159.

Lewis, G. E. \& Anderson, J. K. (1973). The incidence of Brucella canis antibodies in sera of military recruits. Am J Public Health 63, 204-205.

Lucero, N. E. \& Bolpe, J. E. (1998). Buffered plate antigen test as a screening test for diagnosis of human brucellosis. J Clin Microbiol 36, $1425-1427$.

Lucero, N. E., Foglia, L., Ayala, S. M., Gall, D. \& Nielsen, K. (1999). Competitive enzyme immunoassay for diagnosis of human brucellosis. J Clin Microbiol 37, 3245-3248.

Lucero, N. E., Escobar, G. I., Ayala, S. M. \& Lopez, G. (2002). Sensitivity and specificity of an indirect enzyme-linked immunoassay for the diagnosis of Brucella canis infection in dogs. J Med Microbiol 51, 656-660.

McKee, M. A. \& Ballard, J. L. (1999). Mycotic aneurysms of the tibioperoneal arteries. Ann Vasc Surg 13, 188-190.

Monroe, P. W., Silberg, S. L., Morgan, P. M. \& Adess, M. (1975). Seroepidemiological investigation of Brucella canis antibodies in different human population groups. J Clin Microbiol 2, 382-386.

Nielsen, K., Smith, P., Widdison, J., Gall, D., Kelly, L., Kelly, W. \& Nicoletti, P. (2004). Serological relationship between cattle exposed to Brucella abortus, Yersinia enterocolitica O:9 and Escherichia coli O157 : H7. Vet Microbiol 100, 25-30.

Piampiano, P., McLeary, M., Young, L. W. \& Janner, D. (2000). Brucellosis: unusual presentations in two adolescent boys. Pediatr Radiol 30, 355-357.

Polt, S. S. \& Schaefer, J. (1982). A microagglutination test for human Brucella canis antibodies. Am J Clin Pathol 77, 740-744.

Rousseau, P. (1985). Brucella canis infection in a woman with fever of unknown origin. Postgrad Med 78, 249, 253-254, 257.

Schoenemann, J., Lutticken, R. \& Scheibner, E. (1986). Brucella canis infection in man. Dtsch Med Wochenschr 111, 20-22 (in German).

Schoonjans, F., Zalata, A., Depuydt, C. \& Comhaire, F. (1995). MedCalc: a new computer program for medical statistics. Comput Methods Programs Biomed 48, 257-262.

Tosi, M. F. \& Nelson, T. J. (1982). Brucella canis infection in a 17-monthold child successfully treated with moxalactam. J Pediatr 101, 725-727.

Varela-Diaz, V. M. \& Myers, D. M. (1979). Occurrence of antibodies to Brucella canis in rural inhabitants of Corrientes and Neuquén provinces, Argentina. Am J Trop Med Hyg 28, 110-113.

Ying, W., Nguyen, M. Q. \& Jahre, J. A. (1999). Brucella canis endocarditis: case report. Clin Infect Dis 29, 1593-1594. 